\title{
Modeling and analysis of interference in Listen-Before-Talk spectrum access schemes
}

\author{
Alexe E. Leu ${ }^{1}$, Mark McHenry ${ }^{1}$ and Brian L. Mark ${ }^{2, *,+}$ \\ ${ }^{1}$ Shared Spectrum Company, Vienna, Virginia, USA \\ ${ }^{2}$ ECE Department, George Mason University, Fairfax, Virginia, USA
}

\begin{abstract}
SUMMARY
Spectrum measurement studies have shown that substantial portions of the allocated wireless spectrum are highly underutilized. Frequency-agile radios (FARs) have the potential to make opportunistic use of such spectrum holes without causing harmful interference to users of the allocated spectrum. Toward this goal, we develop a framework for modeling the interference caused by FARs employing spectrum access mechanisms based on the simple ListenBefore-Talk (LBT) scheme. Two variations of LBT are considered: individual LBT, whereby the FARs act independently of each other; and collaborative LBT, whereby the FARs communicate with each other in order to more accurately identify the spectrum holes. Our analysis of the LBT scheme reveals the fundamental interdependencies among key system design metrics and provides a basis for analyzing more complex spectrum access methods. In particular, the analysis of LBT provides a lower bound on the capacity gain achievable by FARs employing spectrum-sharing schemes. Our numerical results show that the individual LBT scheme can provide substantial capacity gains, while even more gain can be achieved using the collaborative LBT schemes. Our analysis suggests that much greater gains should be achievable via spectrum access schemes that incorporate location information and/or more sophisticated group behaviors. Copyright (c) 2006 John Wiley \& Sons, Ltd.
\end{abstract}

\section{INTRODUCTION}

In conventional wireless systems, the spectrum is statically allocated among various transmitters located over a geographic coverage area. Recent studies [1,2] suggest that significant portions of the wireless spectrum are highly underutilized. An open research question is whether such 'spectrum holes' can be exploited without causing harmful interference to the primary users, i.e., transmitters and receivers, of the allocated wireless spectrum. In this paper, we model the interference caused to primary users by frequency-agile radios (FARs), which attempt to identify and make use of the spectrum holes by using an access mechanism called Listen-Before-Talk (LBT).

FARs [1], also known as spectrum-agile radios, have the ability to transmit and receive signals on dynamically tunable frequency ranges. Spectrum agility is a key property of the next generation of cognitive radios [3,4]. If a group of FARs could detect the presence of a spectrum hole in a given frequency range, the group would be able to communicate on frequency channels lying within the hole. In this scenario, the FARs must transmit with sufficient power to communicate with each other, but must not cause harmful interference to the primary users. Identification of spectrum holes is made possible by highly sensitive detectors [5].

The FARs may collaborate with each other to identify the spectrum holes. In general, the FAR nodes do not communicate with the primary users. For this reason, the primary nodes are sometimes referred

*Correspondence to: Brian L. Mark, ECE Department, MS 1GS, George Mason University, Fairfax, VA 22030, USA.

${ }^{\dagger}$ E-mail: bmark@gmu.edu

Contract/grant sponsor: National Science Foundation; Grant number: CNS-0520151.

Contract/grant sponsor: Defense Advanced Research Projects Agency and the Air Force; Contract number: FA8750-05-c-0150. 
to as non-cooperative nodes. The non-cooperative nodes may be divided into transmitters and receivers. We refer to the transmitters as primary transmitters and the receivers as victim nodes, to emphasize that the FARs may cause some interference to these receivers. A spectrum-sharing access scheme must ensure that such interference is maintained below a certain tolerable threshold.

The main objective of this paper is to model the interference to the victim nodes caused by a group of FAR nodes employing two variations of the LBT scheme: individual LBT and collaborative LBT. In the individual LBT scheme, each FAR node listens to a given frequency channel for a short time interval called the listen period before attempting to transmit on the channel. If the received signal measurement at the FAR node exceeds a certain threshold, called the detection threshold, the FAR node abandons the current frequency channel and moves on to a different channel. Otherwise, the FAR node transmits on the channel for a short time interval called the transmit period. In the collaborative LBT scheme, if at least one FAR node in a group detects the presence of a signal in a given frequency channel during its listen period, all FAR nodes in the group are alerted to refrain from transmitting on the channel. Collaborative LBT requires the exchange of special alert messages among the FAR nodes. Other variations of LBT are possible, but modeling the interference caused by these simple LBT schemes provides a basis for studying more sophisticated spectrum access schemes [6].

Most of the previous research on resource management for wireless networks has focused on systems in which all of the nodes may cooperate with one another, typically in an infrastructured environment [7]. By contrast, the scenario considered in this paper consists of two subsystems: a non-cooperative system consisting of the set of primary users and a cooperative system consisting of the set of FAR nodes. The goal of the FAR nodes is to make use of the wireless spectrum that is unused by the primary users without causing harmful interference. Thus, an analysis of the interference to the victim nodes caused by the FAR nodes employing a spectrum access a scheme such as LBT is an important step in developing effective spectrum-sharing technologies.

The remainder of the paper is organized as follows. Section 2 describes the LBT schemes in further detail. Section 3 describes the basic model of interference between the FAR nodes employing LBT and the primary users. Analytical expressions for the probability of interference for individual LBT are derived in Section 4. Section 5 extends this analysis to the collaborative LBT scheme. Section 6 presents numerical results showing the performance of the two types of LBT schemes under a wide range of parameter settings. Finally, the paper is concluded in Section 7.

\section{LISTEN-BEFORE-TALK SPECTRUM ACCESS}

Spectrum methods can loosely be classified as cooperative or non-cooperative [6]. Cooperative spectrum access methods seek to provide fair and efficient spectrum usage, typically by means of collision sensing. However, these methods may cause substantial interference to the legacy or non-cooperative users. By contrast, non-cooperative spectrum access methods seek to aviod causing harmful interference to existing users. The Listen-Before-Talk (LBT) scheme is the basis for most non-cooperative spectrum access methods. Hence, a framework for modeling the interference caused by LBT can provide fundamental insights into the behavior of non-cooperative spectrum access in general.

\subsection{Individual LBT}

The Listen-Before-Talk (LBT) algorithm is a simple scheme for an FAR node to access a radio frequency channel opportunistically. Consider a radio frequency channel $c$ centered at the carrier frequency $f_{c}$ and spanning the range $\left[f_{c}-\Delta_{f}, f_{c}+\Delta_{f}\right]$. Thus, the bandwidth of the channel is given by $B_{c}=2 \Delta_{f}$. The LBT scheme consists of two states: (1) listen or off state; and (2) the talk or on state. During the listen state, FAR node does not transmit a signal and estimates the received signal power $R$ in the radio channel $c$, given by

$$
R=\int_{f_{c}-\Delta}^{f_{c}+\Delta} G_{R}(f) \mathrm{d} f
$$

where $G_{R}(f)$ is an estimate of the (one-sided) power spectral density of the received signal. 


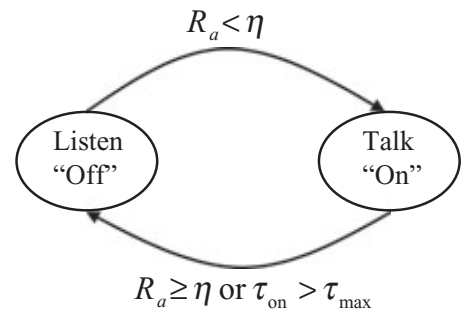

Figure 1. State transition diagram of individual LBT

In the off state, the FAR node also estimates a transmit power level, $s^{*}$, which we refer to as the maximum interference-free transmit power (MIFTP). The MIFTP is defined as the maximum power at which the FAR node can transmit without causing harmful interference to any of the victim nodes. If the received signal power $R$ from the primary transmitter falls below a value $\eta$ called the detection threshold, the FAR node transitions to the on state. Otherwise, the FAR node remains in the off state either for the same channel $c$ or a different frequency channel $c^{\prime}$. For simplicity, our analysis will only consider the case where the FAR node stays in the same channel $c$. During the on state, the FAR node transmits at the MIFTP power level $s^{*}$ for a maximum duration $\tau_{\max }$ and then returns to the off state. During the off state, the FAR node continues to listen to the channel and returns to the off state if $R<\eta$. Figure 1 illustrates the LBT algorithm by means of a state transition diagram.

In this paper, we shall ignore the channel contention among FAR nodes accessing the same channel. Such contention can be resolved using a suitable medium access control (MAC) protocol. Thus, the maximum transmission duty cycle of an FAR node on a given channel is given by

$$
d_{\max }=\frac{\tau_{\text {on }}}{\tau_{\text {on }}+\tau_{\text {off }}}
$$

where $\tau_{\text {off }}$ is the average duration in the off state. Clearly, the amount of interference caused to the victim nodes by an FAR node performing LBT depends on the values of the key parameters $\eta$, $\tau_{\text {on }}$, and the MIFTP $s^{*}$. The interdependencies among these parameters are analyzed in Section 4.

\subsection{Collaborative LBT}

Collaborative LBT extends the individual LBT scheme by allowing a group of FAR nodes to share information gathered during the listen period with each other via alert messages. In collaborative LBT, each FAR node in the group executes the individual LBT algorithm for a given frequency channel $c$ as discussed earlier with the following modification. If at least one FAR node in the group detects the presence of a signal from a primary transmitter, then all of the FAR nodes in the group turn off and revert to the listen state. Otherwise, the channel $c$ is then accessed by the group of FAR nodes using a MAC protocol. We shall not concern ourselves with the details of the MAC protocol used among the FAR nodes. We shall simply choose a particular FAR node in the group and assume that it can transmit at MIFTP during its talk period without causing interference to the other FAR nodes.

\section{INTERFERENCE MODEL}

In this section, we develop a simple model to characterize the interference caused by an FAR node to a victim receiver.

\subsection{Interference scenario}

Consider a scenario consisting of three nodes: a primary transmitter $p$, a victim receiver $v$, and a frequency-agile node denoted by $a$, as shown in Figure 2. The transmitter $p$ transmits on frequency 


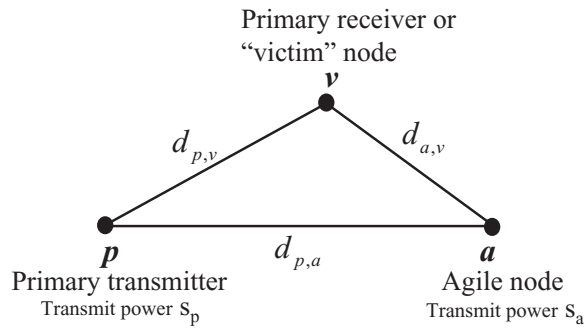

Figure 2. Interference scenario

channel $c$, while node $a$ performs LBT on the channel. The receiver $v$ expects to receive the transmissions of node $p$. However, node $a$ may cause interference to node $v$ during the talk state of LBT.

Denote the geographic locations of the nodes by $\left(x_{i}, y_{i}\right)$, where $i \in\{a, p, v\}$. Let $d_{i j}$ and $L_{i j}$ denote, respectively, the distance and propagation loss from node $i$ to node $j$, where $i, j \in\{a, p, v\}$. Clearly, the propagation loss $L_{i j}$ depends on the propagation distance $d_{i j}$. We model the primary transmitter $p$ and the FAR node $a$ as on-off sources. In the off states, both sources do not transmit signals. In the on states, node $p$ transmits with power $s_{p}$, while node $a$ transmits with power $s_{a}$ in the off state. The received signal power from the primary transmitter $p$ during its on state at nodes $a$ and $v$ can be expressed as follows:

$$
\begin{aligned}
& R_{a}=s_{p}-L_{p, a} \\
& R_{v}=s_{p}-L_{p, v}
\end{aligned}
$$

Similarly, we represent the interference signal power from node $a$ received at node $v$ is given by

$$
I_{v}=s_{a}-L_{a, v}
$$

Mathematically, all of the quantities in (3)-(5) are stochastic processes, although the time parameter $t$ has been suppressed in the above equations to emphasize the steady-state condition.

For successful reception of the primary signals from node $p$ at node $v$, certain conditions must be satisfied. Let $r_{\min }$ denote the minimum received signal level required by the primary receiver $v$ from the primary transmitter $p$. Denote by $i_{\max }$ the maximum allowable interference that node $v$ can tolerate. Let $E_{a}^{(\text {on })}$ and $E_{p}^{(\text {on })}$ denote, respectively, the events that nodes $a$ and node $p$ are in the on state. Then the event that node $a$ causes harmful interference to node $v$, i.e., the interference event, can be expressed as follows:

$$
E_{\text {int }}=\left\{R_{v} \geq r_{\min }, E_{p}^{\text {(on) }}\right\} \cap\left\{I_{v} \geq i_{\text {max }}, E_{a}^{\text {(on) }}\right\}
$$

Thus, the interference event occurs if and only if the primary transmitter $p$ is on and the received signal at node $v$ from $p$ exceeds $r_{\min }$ and node $a$ is on and the received interference signal at node $v$ from $a$ exceeds $i_{\max }$.

\subsection{Propagation model}

The signal propagation loss is modeled as a sum of three components [8]: path loss, log-normal shadowing noise, and Rayleigh fast fading. We shall assume that the fast fading can be eliminated by averaging the received signal measurements over time [9]. Thus, the propagation loss between nodes $i$ and $j$ can be expressed as

$$
L_{i j}=D_{i j}+W_{i j}
$$

where $D_{i j}$ and $W_{i j}$ represent the path loss and shadowing noise, respectively. In general, the path loss is a function several variables: 
- the locations of nodes $i$ and $j$ and the nature of the geographic terrain between them;

- the transmitter antenna height $h_{i}^{(t)}$;

- the receiver antenna height $h_{j}^{(r)}$;

- the carrier frequency $f_{c}$;

- the antenna polarization $o_{i}$ (horizontal or vertical).

The shadowing component $W_{i j}$ is assumed to be zero-mean white Gaussian noise process with variance $\sigma_{i j}^{2}$ and independent of the path loss $D_{i j}$.

The path loss $D_{i j}$ component can be modeled using the empirical propagation model (EPM-73) [12] or more complicated propagation prediction models such as the Longley-Rice model [13-14] or the TIREM (Terrain Integrated Rough Earth Model) [15]. For simplicity, we shall assume the EPM-73 model in this paper. According to the EPM-73 model, the mean path loss for a given terrain type (hilly, swamp, etc.) depends only on the distance between the two nodes. Hence, we can write

$$
D_{i j}=g\left(d_{i j}, h_{i}^{(t)}, h_{j}^{(r)}, f_{c}, o_{i}\right)
$$

where the function $g(\cdot)$ is given in [12]. For notational convenience, we shall simply write $D_{i j}=g\left(d_{i j}\right)$ and suppress the dependence of the path loss on the remaining four parameters. Since the function $g(\cdot)$ is invertible, the distance can be expressed in terms of the path loss by

$$
d_{i j}=g^{-1}\left(D_{i j}\right)
$$

\section{ANALYSIS OF INDIVIDUAL LBT}

In this section, we derive expressions for the interference probability for the individual LBT scheme based on the interference model discussed in Section 3. We also derive several other key system parameters and discuss their performance impacts.

\subsection{Outage probability and coverage distance}

The outage probability (of node $v$ ) is defined by

$$
P_{\text {out }} \triangleq P\left\{R_{v}<r_{\min } \mid E_{p}^{(\text {on })}\right\}
$$

Hence,

$$
P\left\{R_{v} \geq r_{\min } \mid E_{p}^{(\text {on })}\right\}=\left(1-P_{\text {out }}\right)
$$

Assuming the EPM-73 propagation path loss model, from (4), (7), and (8), we can write

$$
R_{v}=s_{p}-g\left(d_{p, v}\right)+W_{p, v}
$$

Therefore,

$$
P_{\text {out }}=1-Q\left(\frac{r_{\min }+g\left(d_{p, v}\right)-s_{p}}{\sigma_{p, v}}\right)
$$

Closely related to the outage probability is the coverage distance, $d_{\mathrm{cov}}$, defined as the maximum distance between the primary transmitter and the victim node such that the outage probability does not exceed a value $\varepsilon_{\text {out }}$ :

$$
d_{\text {cov }}\left(\varepsilon_{\text {out }}\right) \triangleq \max \left\{d_{p, v}: P_{\text {out }} \leq \varepsilon_{\text {out }}\right\}
$$

From (13) and (12), we can write

$$
d_{\mathrm{cov}}=g^{-1}\left(s_{p}-r_{\min }+\sigma_{p, v} Q^{-1}\left(1-\varepsilon_{\mathrm{out}}\right)\right)
$$


We define the detection probability, $P_{\text {det }}$ at the FAR node as the probability that the received signal from the primary transmitter is greater than or equal to a given threshold $\eta$. That is, $P_{\text {det }}$ is given by

$$
P_{\text {det }}=P\left\{R_{a} \geq \eta\right\}=1-Q\left(\frac{s_{p}-g\left(d_{p, a}\right)-\eta}{\sigma_{p, v}}\right)
$$

The detection distance, $d_{\mathrm{det}}$, at the FAR node is defined as the maximum distance between the FAR node and the primary transmitter such that the probability of detection at the FAR node exceeds a value $\varepsilon_{\text {det }}$ :

$$
d_{\mathrm{det}}\left(\varepsilon_{\mathrm{det}}\right) \triangleq \max \left\{d_{a, p}: P_{\mathrm{det}} \geq \varepsilon_{\mathrm{det}}\right\}
$$

Assuming that the function $g^{-1}(\cdot)$ is smooth, from (16) and (17), we have

$$
d_{\text {det }}\left(\varepsilon_{\text {det }}\right)=g^{-1}\left(s_{p}-\eta-\sigma_{p, v} Q^{-1}\left(1-\varepsilon_{\text {det }}\right)\right)
$$

The detection distance decreases monotonically with the bound on the detection probability, $\varepsilon_{\text {out }}$, which we will show numerically in Section 6.

\subsection{Interference probability and distance}

The signal power received by node $v$ from node $p$ in the on state is independent of the interference power it receives from node $a$ in the on state. Therefore, from (6) we have

$$
P_{\text {int }}=P\left\{R_{v} \geq r_{\min }, E_{p}^{(\text {on })}\right\} \cdot P\left\{I_{v} \leq i_{\max }, E_{a}^{(\text {on })}\right\}
$$

From (5), (7), and (8), we can write

$$
I_{v}=s_{a}-g\left(d_{a, v}\right)+W_{a, v}
$$

Therefore,

$$
P\left\{I_{v} \leq i_{\max } \mid E_{a}^{(o n)}\right\}=Q\left(\frac{i_{\max }+g\left(d_{a, v}\right)-s_{a}}{\sigma_{a, v}}\right)
$$

where $Q(x) \triangleq \frac{1}{\sqrt{2 \pi}} \int_{x}^{\infty} e^{-t^{2} / 2} \mathrm{~d} t$ is the standard Q-function. Using (19), (11), and (21), we obtain the following expression for the interference probability:

$$
P_{\text {int }}=\left(1-P_{\text {out }}\right) \cdot P\left(E_{p}^{(\text {on })}\right) P\left(E_{a}^{(\text {on })}\right) \cdot\left(\frac{i_{\max }+g\left(d_{a, v}\right)-s_{a}}{\sigma_{a, v}}\right)
$$

From (22), we can obtain an expression for the transmit signal power of the FAR node in terms of the interference and outage probabilities as follows:

$$
s_{a}=\sigma_{a, v} Q^{-1}\left(1-\frac{P_{\text {int }}}{\left(1-P_{\text {out }}\right) P\left(E_{p}^{(\text {on })}\right) P\left(E_{a}^{(\text {on })}\right)}\right)+i_{\max }+g\left(d_{a, v}\right)
$$

To proceed further with the interference analysis of LBT, we make the following worst-case assumptions about the on-off source behavior of the primary transmitter and the FAR node:

- The primary transmitter is always in the on state, i.e., $P\left(E_{p}^{(o n)}\right)=1$.

- The FAR node is in the on state if and only if the received signal from the primary transmitter falls below the threshold $\eta$.

With reference to the LBT state diagram of Figure 1, the second assumption is tantamount to the assumption that $\tau_{\max }=\infty$. In this case, we have that

$$
P\left(E_{a}^{(\text {on })}\right)=1-P_{\text {det }}
$$

Under the above two assumptions, the duty cycle of the FAR node executing LBT is given by [2]

$$
d_{a}=P\left(E_{a}^{(\mathrm{on})}\right)=1-P_{\mathrm{det}}
$$


Let $\varepsilon_{\text {int }}$ be the maximum interference probability that can be tolerated by the victim node $v$. Under these assumptions, we define the interference distance, $d_{\text {int }}\left(\varepsilon_{\text {int }}\right)$, as the minimum permissible distance between the FAR node $a$ and the victim node $v$ such that the interference probability does not exceed $\varepsilon_{\text {int }}$.

$$
d_{\text {int }}\left(\varepsilon_{\text {int }}\right) \triangleq \min \left\{d_{a, v}: P_{\text {int }} \leq \varepsilon_{\text {int }}\right\}
$$

From (22), we obtain the following expression for the interference distance:

$$
d_{\text {int }}=g^{-1}\left(s_{a}-i_{\max }-\sigma_{a, v} Q^{-1}\left(1-\frac{\varepsilon_{\text {int }}}{\left(1-P_{\text {out }}\right)\left(1-P_{\text {det }}\right)}\right)\right)
$$

\subsection{Avoidance of harmful interference}

The victim node is said to suffer harmful interference from the FAR node under the following conditions:

$$
P_{\text {out }} \leq \varepsilon_{\text {out }}, P_{\text {det }} \leq \varepsilon_{\text {det }}, P_{\text {int }}>\varepsilon_{\text {int }}
$$

The first condition states that the outage probability of the victim node should not exceed the threshold $\varepsilon_{\text {out }}$. If the outage probability exceeds $\varepsilon_{\text {out }}$, the victim node is oblivious to interference from the FAR node. The second condition requires the detection probability not to exceed the detection probability threshold $\varepsilon_{\mathrm{det}}$, which implies that the duty cycle of the FAR node would be at least $1-\varepsilon_{\mathrm{det}}$. Finally, the third condition requires the probability of interference to be greater than a threshold $\varepsilon_{\text {int. }}$.

From (22), we can derive an expression for the maximum power at which the FAR node can transmit while avoiding harmful interference to node $v$ for a given internodal distance $d_{a, v}$ :

$$
s_{a}^{*}=i_{\max }+g\left(d_{a, v}\right)+\sigma_{a, v} Q^{-1}\left(1-\frac{\varepsilon_{\text {int }}}{\left(1-P_{\text {out }}\right)\left(1-P_{\text {det }}\right)}\right)
$$

We call $s_{a}^{*}$ the maximum interference-free transmit power (MIFTP). The MIFTP plays a key role in LBT-based spectrum access.

Assume that the transmit powers of the FAR node and the primary transmitter are fixed at $s_{a}$ and $s_{p}$, respectively. The following proposition gives a simple sufficient condition for the victim node not to suffer harmful interference in terms of an inequality relating the coverage distance, the detection distance, and the interference distance.

Proposition 1: Suppose that the first two conditions in (26) hold, i.e.,

$$
P_{\text {out }} \leq \varepsilon_{\text {out }} \text { and } P_{\text {det }} \leq \varepsilon_{\text {det }}
$$

Then the victim node does not suffer harmful interference if

$$
d_{\text {int }} \leq d_{\text {det }}-d_{\text {cov }}
$$

Proof: By definition, the conditions $P_{\text {out }} \leq \varepsilon_{\text {out }}$ and $P_{\text {det }} \leq \alpha$ imply

$$
d_{p, v} \leq d_{\mathrm{cov}} \text { and } d_{a, p} \geq d_{\mathrm{det}}
$$

respectively. Using the triangle inequality, we have

$$
d_{a, v} \leq d_{a, p}-d_{p, v}
$$

From (30) and (31), we have

$$
d_{a, v} \geq d_{\mathrm{det}}-d_{\mathrm{cov}} \geq d_{\mathrm{int}}
$$

which implies that

$$
P_{\text {int }} \leq \varepsilon_{\text {int }}
$$

Hence, the harmful interference condition (26) does not hold.

Clearly, the converse of Proposition (1) does not hold in general. The FAR node can always be positioned sufficiently far from the victim and primary nodes in order to avoid harmful interference. A 


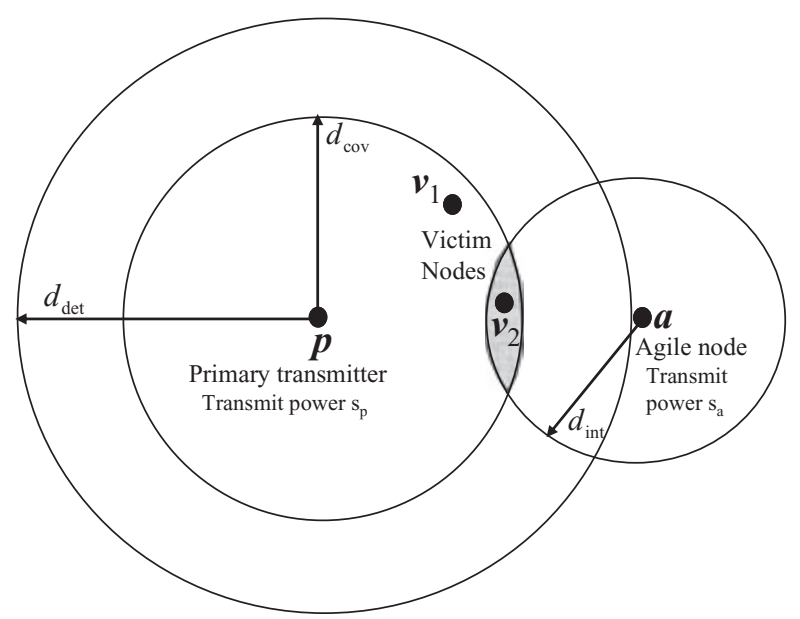

Figure 3. Interference condition

graphical illustration of Proposition 1 is given in Figure 3. In Figure 3, we have that both victim nodes $v_{1}$ and $v_{2}$ are within a distance $d_{\text {cov }}$ from the primary transmitter. Hence, the outage probabilities of the victim nodes do not exceed $\varepsilon_{\text {out }}$. The FAR node $a$ lies just outside the distance $d_{\text {det }}$ from the primary transmitter. Hence, the detection probability does not exceed $\varepsilon_{\operatorname{det}}$.

Observe that although node $v_{1}$ lies beyond the detection distance, $d_{\text {det }}$ from the agile node $a$, the node $v_{2}$ does not. Hence, node $v_{2}$ suffers from harmful interference, i.e., the interference probability experienced by node $v_{2}$ exceeds $\varepsilon_{\text {int }}$. Moreover, any victim node lying within the shaded region would suffer harmful interference. The shaded region can be reduced by lowering the transmit power, $s_{a}$. The absence of the shaded region corresponds to the non-interference condition (29). If this condition holds, no victim node can suffer harmful interference from the FAR node.

\section{ANALYSIS OF COLLABORATIVE LBT}

In collaborative LBT, we consider a group of $n$ FAR nodes $\mathscr{G}=\left\{a_{1}, \ldots, a_{n}\right\}$. Each of these nodes executes the individual LBT algorithm with the proviso that if at least one node in the group detects a signal from the primary transmitter, all nodes in the group turn to the off state. Otherwise, each node is in the on state transmitting at a certain power level. Let $R_{a_{i}}$ denote the signal power from the primary transmitter that is received at FAR node $a_{i}[3]$ :

$$
R_{a_{i}}=s_{p}+L_{p, a_{i}}
$$

Then the probability that the FAR nodes are all in the on state is given by

$$
P\left(E_{a}^{\text {(on })}\right)=P\left[\bigcap_{i=1}^{n}\left\{R_{a_{i}}<\eta\right\}\right]
$$

where $\eta$ is the detection threshold. Assuming that the received signal strengths are independent,

$$
\begin{aligned}
P\left(E_{a}^{(\text {on })}\right) & =\prod_{i=1}^{n} P\left\{R_{a_{i}}<\eta\right\} \\
& =\prod_{i=1}^{n} Q\left[\frac{s_{p}-\eta-g\left(d_{p, a_{i}}\right)}{\sigma_{p, a_{i}}}\right]
\end{aligned}
$$


Let $\alpha$ be the probability that all the FAR nodes are in the off state, i.e.,

$$
a=1-P\left(E_{a}^{\text {(on) })}\right)=1-\prod_{i=1}^{n} Q\left[\frac{s_{p}-\eta-g\left(d_{p, a_{i}}\right)}{\sigma_{p, a_{i}}}\right]
$$

If all nodes in $\mathscr{G}$ are equidistant from node $p$ and the propagation characteristics from $p$ to the FAR nodes are homogeneous, then

$$
\alpha=1-Q^{n}\left[\frac{s_{p}-\eta-g\left(d_{p, a}\right)}{\sigma_{p, a}}\right]
$$

where $d_{p, a}$ is the common distance from node $p$ to each FAR node, and $\sigma_{p, a}$ is the common shadowing standard deviation. Under the assumption of homogeneity of the FAR nodes with respect to node $p$, we can define a detection distance analogous to (18), with $\alpha$ given by (38). Similarly, we can define an interference distance analogous to (25) and an MIFTP analogous to (27).

\section{NUMERICAL RESULTS}

In this section, we present numerical results showing performance metrics for individual and collaborative LBT over a range of parameter values using the expressions derived in Sections 4 and 5 . The results provide insight into the performance impacts of the various key system parameters in LBT spectrum access.

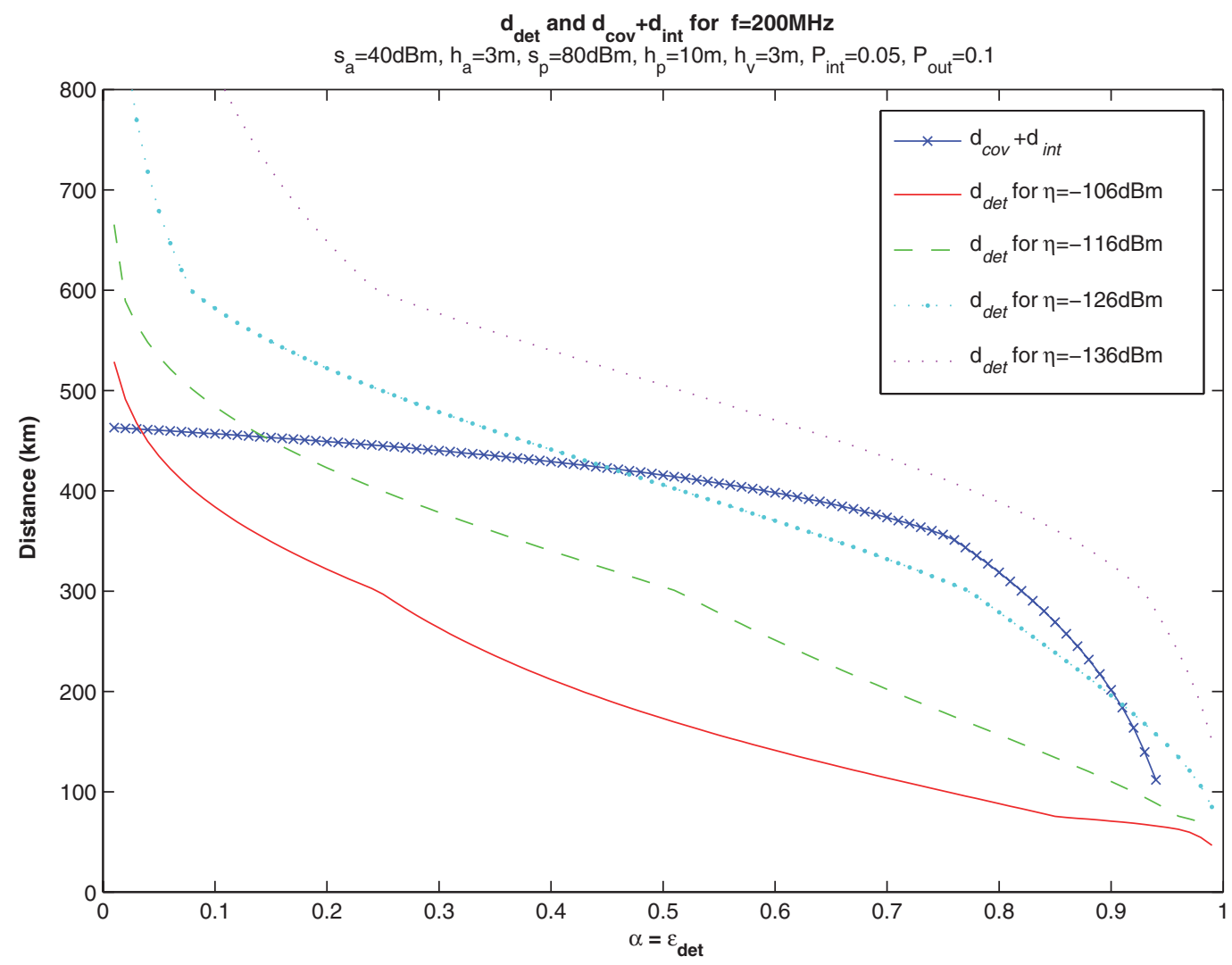

Figure 4. Interference, coverage, and detection distances for $f_{c}=200 \mathrm{MHz}$ 


\subsection{Interference, coverage, and detection distances}

Figures 4 and 5 show plots of the detection distance $d_{\operatorname{det}}$ as a function of the maximum detection probability $\varepsilon_{\text {det }}$ for different values of the detection threshold $\eta$ in individual LBT. Figure 4 shows curves obtained for a carrier frequency of $f_{c}=200 \mathrm{MHz}$, while Fig. 5 corresponds to $f_{c}=2.4 \mathrm{GHz}$. The frequency $f_{c}=200 \mathrm{MHz}$ lies within the transmission band of television towers, while $f_{c}=2.4 \mathrm{GHz}$ lies within the transmission band of MMDS (Multipoint Microwave Distribution System) systems. The detection distance curves were obtained using (18). The graphs also show the sum of the interference and coverage distances, i.e., $d_{\text {int }}+d_{\text {cov }}$ as a function of the detection probability $\varepsilon_{\text {det }}$. The values of $d_{\text {int }}$ and $d_{\text {cov }}$ were calculated using (25) and (15), respectively. The other system parameters of interest are set as follows:

- FAR transmitter power $s_{a}=40 \mathrm{dBm}$;

- FAR and victim node receiver antenna heights: $h_{a}^{(r)}=h_{v}^{(r)}=3 \mathrm{~m}$

- FAR and primary transmitter antenna heights: $h_{a}^{(t)}=h_{p}^{(t)}=10 \mathrm{~m}$

- primary transmitter power $s_{p}=80 \mathrm{dBm}$;

- interference probability $P_{\text {int }}=0.01$;

- maximum outage probability $P_{\text {out }}=0.1$.

The detection threshold ranges from $\eta=-136 \mathrm{dBm}$ to $\eta=-106 \mathrm{dBm}$.

Observe that both the detection distance and the interference distance are monotonically decreasing functions of the detection probability. To avoid harmful interference, the detection threshold $\eta$ should be chosen such that the non-interference condition (29) holds over the entire range of values of $\varepsilon_{\text {int, }}$ since we have no prior knowledge of the detection probability. With respect to Figures 4 and 5, the non-interference condition requires the $d_{\text {det }}$ curve to lie above the $\left(d_{\text {int }}+d_{\text {cov }}\right)$ curve for all values of $\alpha$.

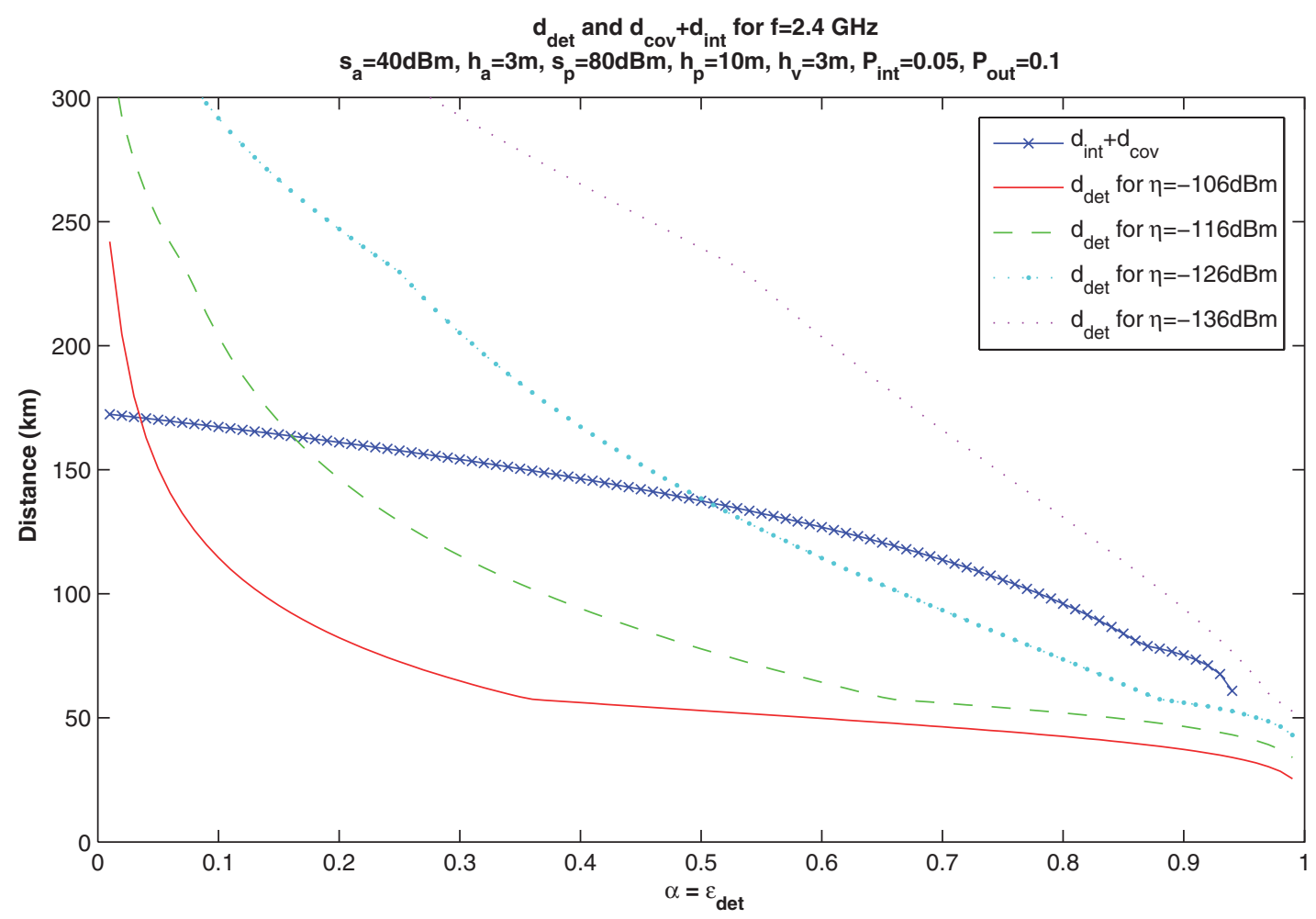

Figure 5. Interference, coverage, and detection distances for $f_{c}=2.4 \mathrm{GHz}$ 
We observe that the non-interference condition holds when the detection threshold satisfies $\eta=$ $-136 \mathrm{~dB}$ for both carrier frequencies. This implies a lower bound on the sensitivity of the detector of the FAR node to ensure that it does not cause harmful interference to the victim node. Note that at the higher carrier frequency $f_{c}=2.4 \mathrm{GHz}$, the interference, detection, and coverage curves are all significantly lower than at $f_{c}=200 \mathrm{MHz}$. When $f_{c}=2.4 \mathrm{GHz}$, the peak value of $d_{\text {int }}+d_{\text {cov }}$ is about $175 \mathrm{~km}$, whereas the corresponding value at $f_{c}=200 \mathrm{MHz}$ is about $460 \mathrm{~km}$.

\subsection{MIFTP versus detection threshold}

Figure 6 show curves of the MIFTP as a function of the detection threshold $\eta$ for the carrier frequencies $f_{c}=200 \mathrm{MHz}$ and $f_{c}=2.4 \mathrm{GHz}$, respectively. Observe that the MIFTP curve for $f_{c}=2 \mathrm{GHz}$ lies below the curve for $f_{c}=200 \mathrm{MHz}$. Thus, at lower carrier frequencies the FAR node can transmit with higher signal power without causing harmful interference to the victim receiver.

\subsection{MIFTP versus interference probability}

Figures 7 and 8 show surfaces of the MIFTP as a function of the detection threshold $\eta$ and the interference probability $P_{\text {int }}$ for the carrier frequencies $f_{c}=200 \mathrm{MHz}$ and $f_{c}=2.4 \mathrm{GHz}$, respectively. We observe that the surface corresponding to $f_{c}=2 \mathrm{GHz}$ lies below the surface for $f_{c}=200 \mathrm{MHz}$. The decreasing trend of the MIFTP with respect to the detection threshold is similar at both carrier frequencies.

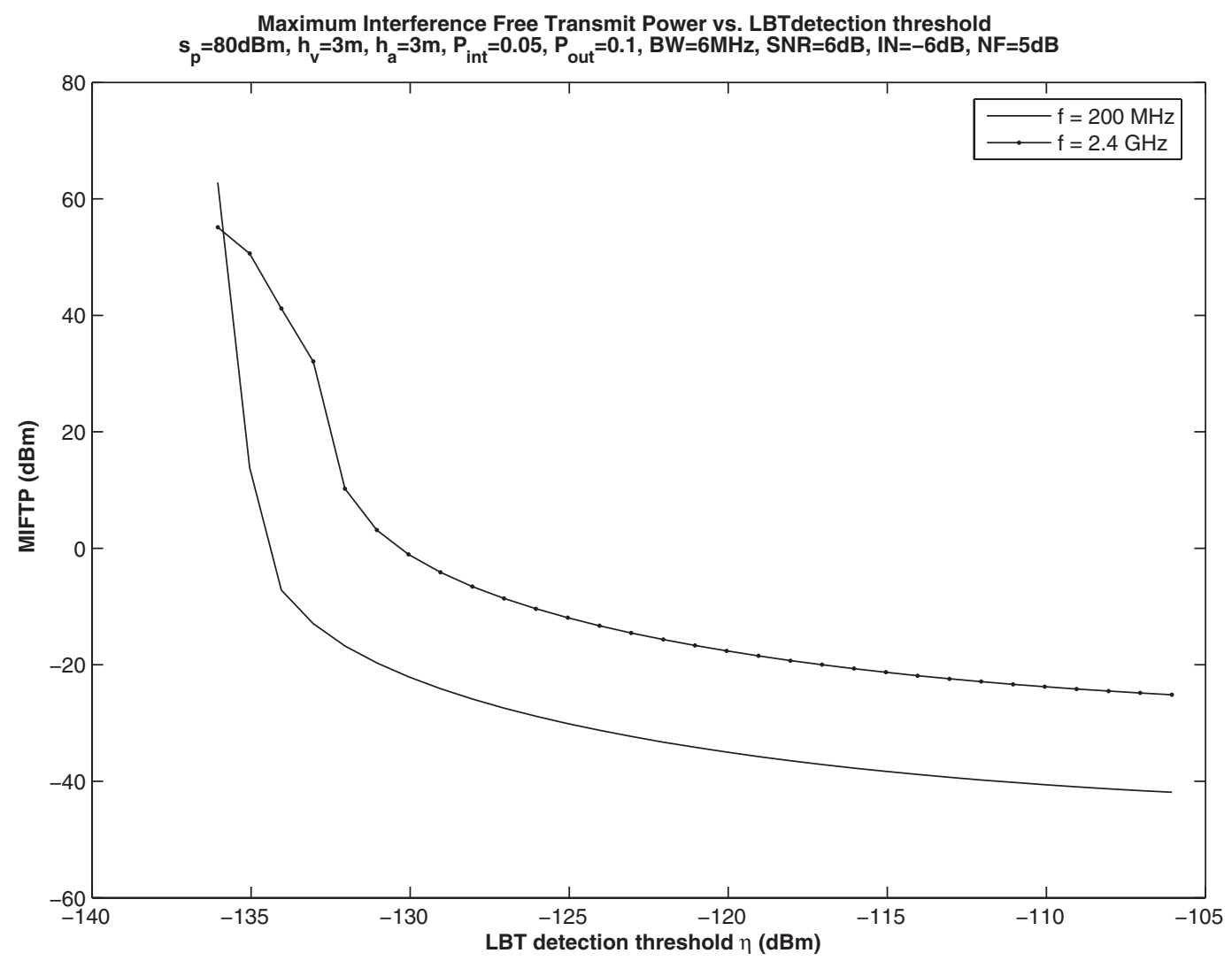

Figure 6. MIFTP vs. LBT detection threshold $\eta$ 


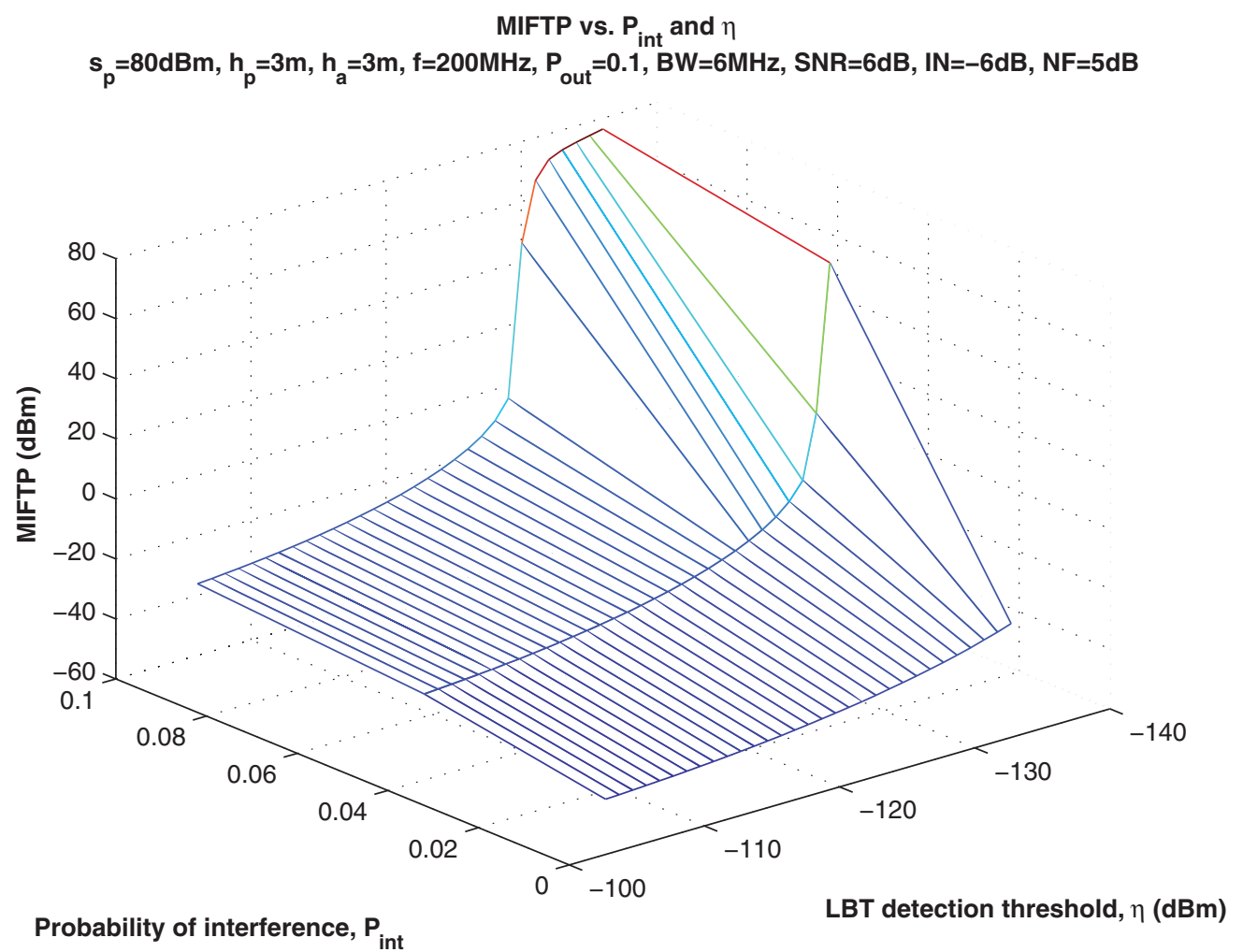

Figure 7. MIFTP versus detection threshold and interference probability for $f_{c}=200 \mathrm{MHz}$

\subsection{MIFTP versus FAR transmitter antenna height}

Figures 9 and 10 show surfaces of the MIFTP as a functions of the detection threshold $\eta$ and the FAR transmitter antenna height $h_{a}^{(t)}$ for $f_{c}=200 \mathrm{MHz}$ and $f_{c}=2 \mathrm{GHz}$, respectively. At both carrier frequencies, the MIFTP increases linearly with increasing antenna height. Note that the MIFTP surface at the higher carrier frequency has a larger slope with respect to the FAR transmitter antenna height compared to the MIFTP surface at the lower frequency. In fact, the two surfaces, cross each other at an antenna height of approximately $150 \mathrm{~m}$.

\subsection{MIFTP versus carrier frequency}

Figure 11 shows an MIFTP surface as a function of the detection threshold $\eta$ and the carrier frequency $f_{c}$. The MIFTP surface is a decreasing function of $\eta$, but is not a monotonic function of $f_{c}$. As can be observed in Figure 11, the MIFTP decreases roughly linearly as $f_{c}$ is increased from $0 \mathrm{~Hz}$ to about $500 \mathrm{MHz}$. Then the MIFTP increases linearly as $f_{c}$ increases from $500 \mathrm{MHz}$ to a maximum value of $65 \mathrm{MHz}$ when $f_{c}$ is approximately $1200 \mathrm{~Hz}$. Finally, the MIFTP decreases linearly for $f_{c}$ larger than $1200 \mathrm{~Hz}$.

\subsection{Collaborative LBT}

Figures 12 and 13 show MIFTP surfaces for collaborative LBT under homogeneous scenarios for carrier frequencies $f_{c}=200 \mathrm{MHz}$ and $f_{c}=2 \mathrm{GHz}$, respectively. We see that the MIFTP increases with the number of FAR nodes in the group. 


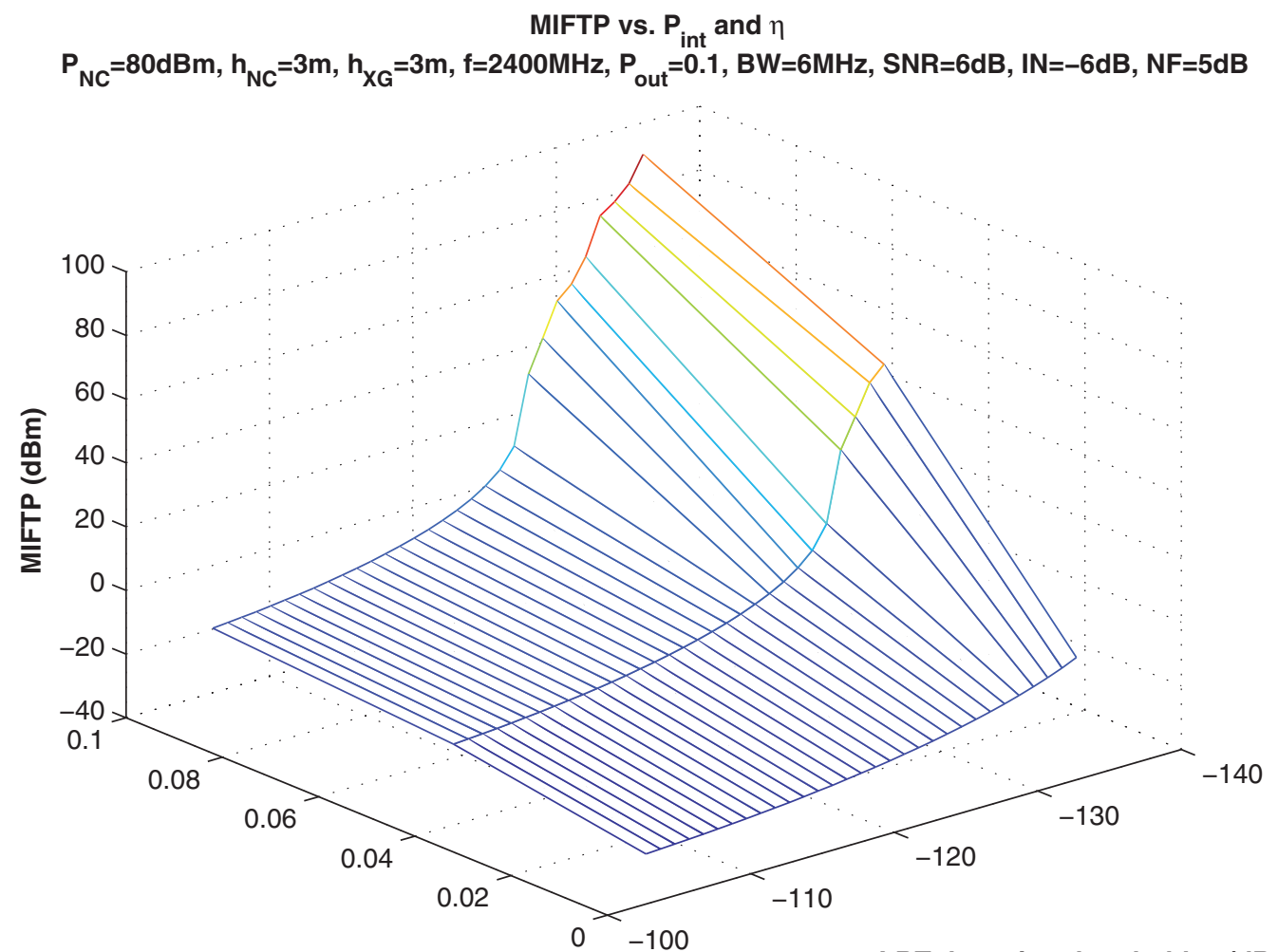

Probability of interference, $P_{\text {int }}$

LBT detection threshold, $\eta(\mathrm{dBm})$

Figure 8. MIFTP versus detection threshold and interference probability for $f_{c}=2.4 \mathrm{GHz}$

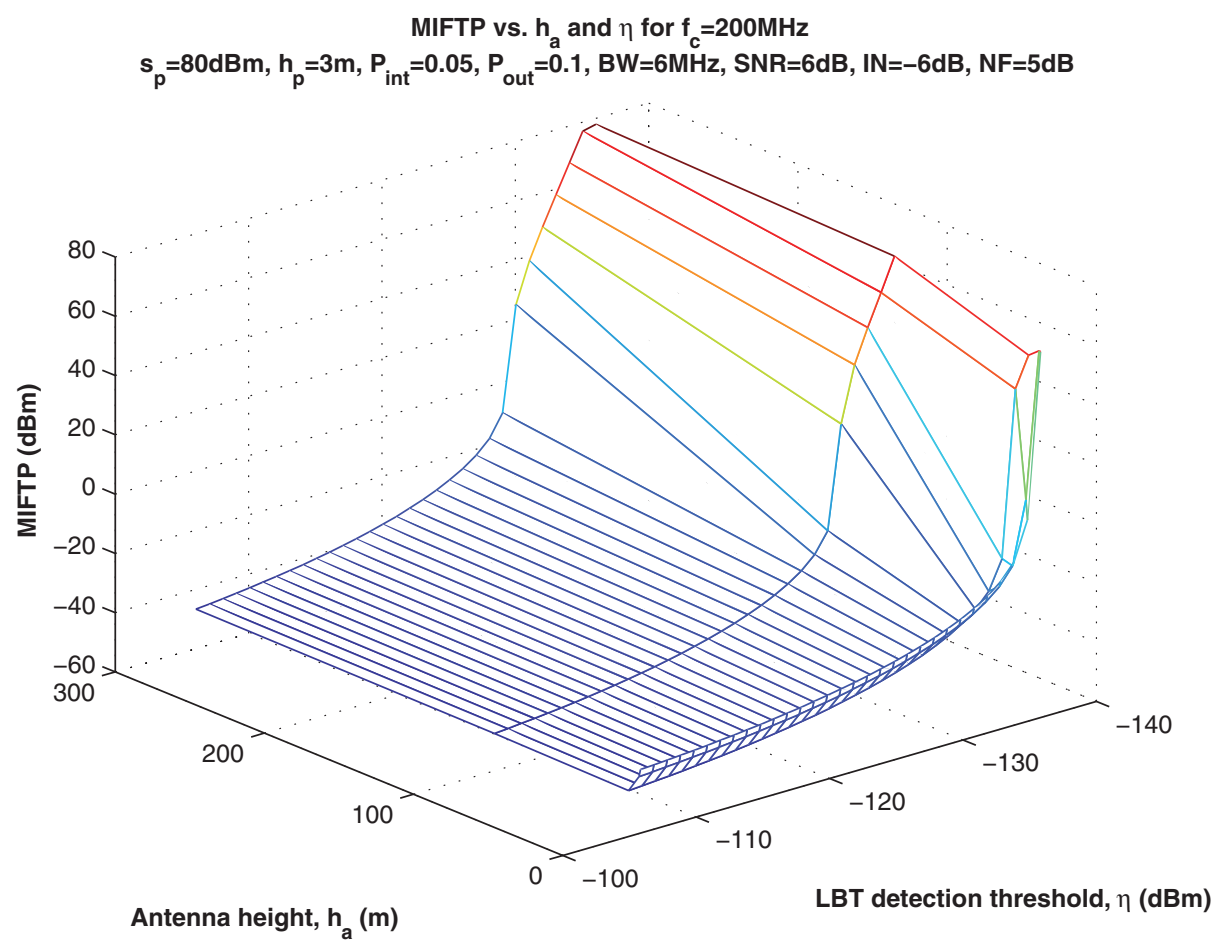

Figure 9. MIFTP versus detection threshold and FAR transmitter antenna height for $f_{c}=200 \mathrm{MHz}$ 


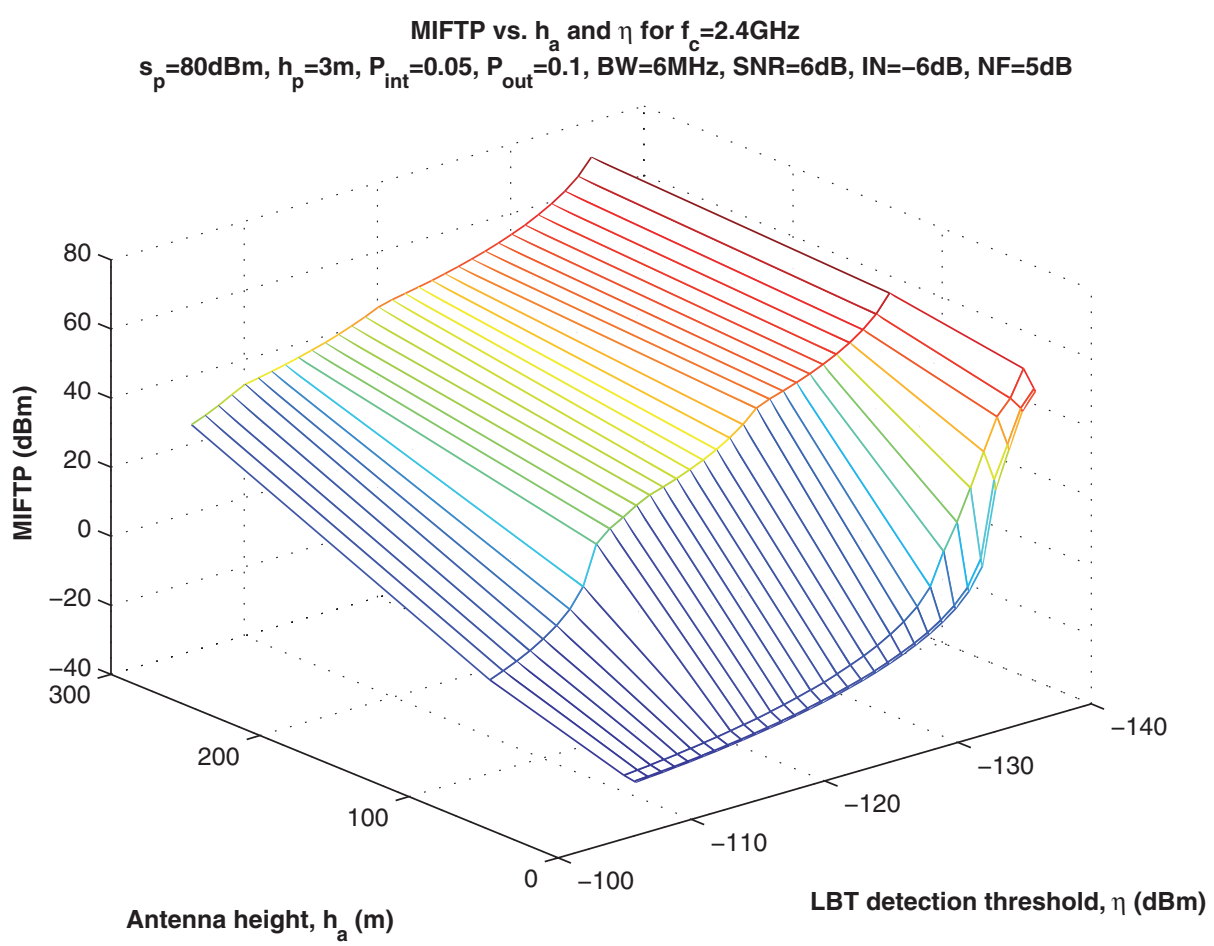

Figure 10. MIFTP versus detection threshold and FAR transmitter antenna height for $f_{c}=2.4 \mathrm{GHz}$

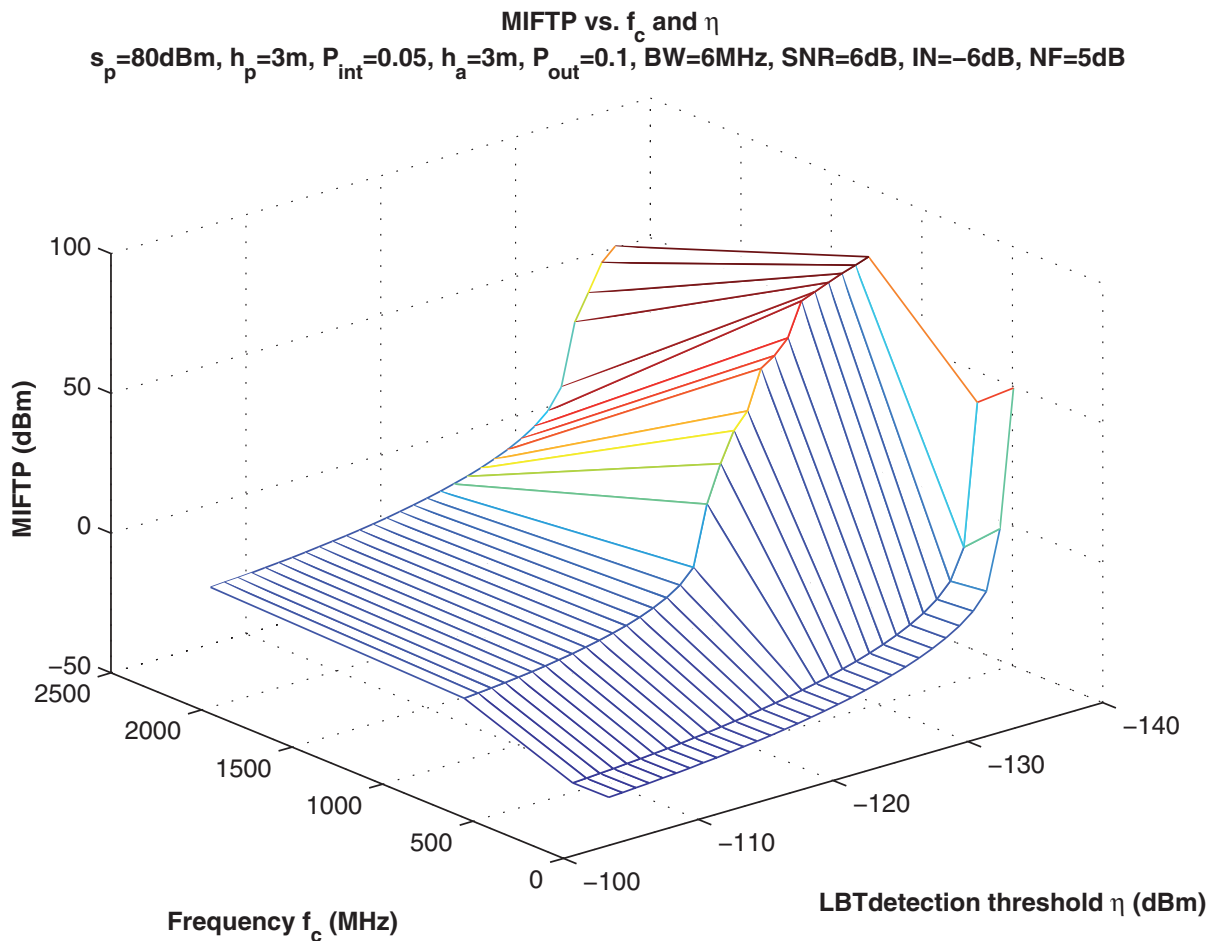

Figure 11. MIFTP versus carrier frequency and detection threshold 


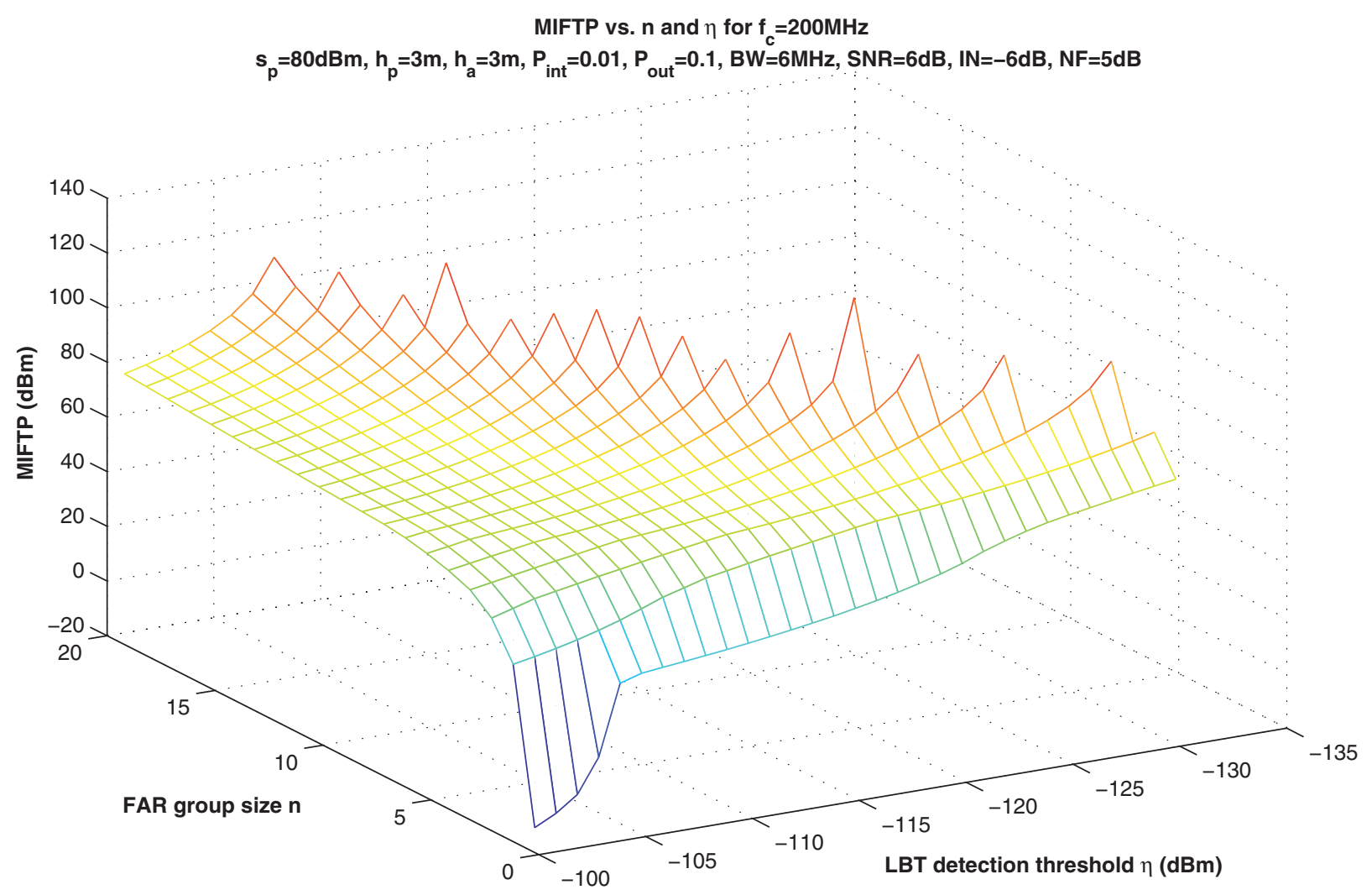

Figure 12. MIFTP versus detection threshold and FAR group size for collaborative sensing at $f_{c}=200 \mathrm{MHz}$

\section{CONCLUSION}

We developed a framework to evaluate the performance of the basic Listen-Before-Talk (LBT) scheme and an extension of LBT called collaborative LBT that allows a group of FAR radios to collaborate. We introduced several important performance metrics including detection distance, interference distance, and maximum interference-free transmit power. These metrics can provide the basis for evaluating any spectrum access method. We derived expressions to evaluate these metrics with respect to the individual and collaborative LBT schemes and presented numerical results illustrating the relationships among them.

The numerical results show that the simple LBT can be used to harvest unused spectrum without causing harmful interference to existing users. The collaborative LBT scheme provides significantly higher spectrum capacity gains than individual LBT. The poorer performance of individual LBT is due to the fact that the nodes act without any knowledge about the location of the primary transmitter and the victim nodes. The collaborative LBT scheme provides additional information about the location of the primary implicitly through the use of alert messages. Spectrum-sharing schemes employing more sophisticated collaborative behaviors should be able to harvest substantially more spectrum. In ongoing work, we are applying the analysis techniques developed in this paper to evaluate the performance of spectrum access with more complex group behaviors. The formulas derived in this paper for the performance metrics were based on the EPM-73 propagation model, which has a relatively simple closed form. In future work, we plan to evaluate the performance of spectrum access using more sophisticated models such as the TIREM and Longley-Rice models, together with spectrum measurement data obtained through drive test experiments. 


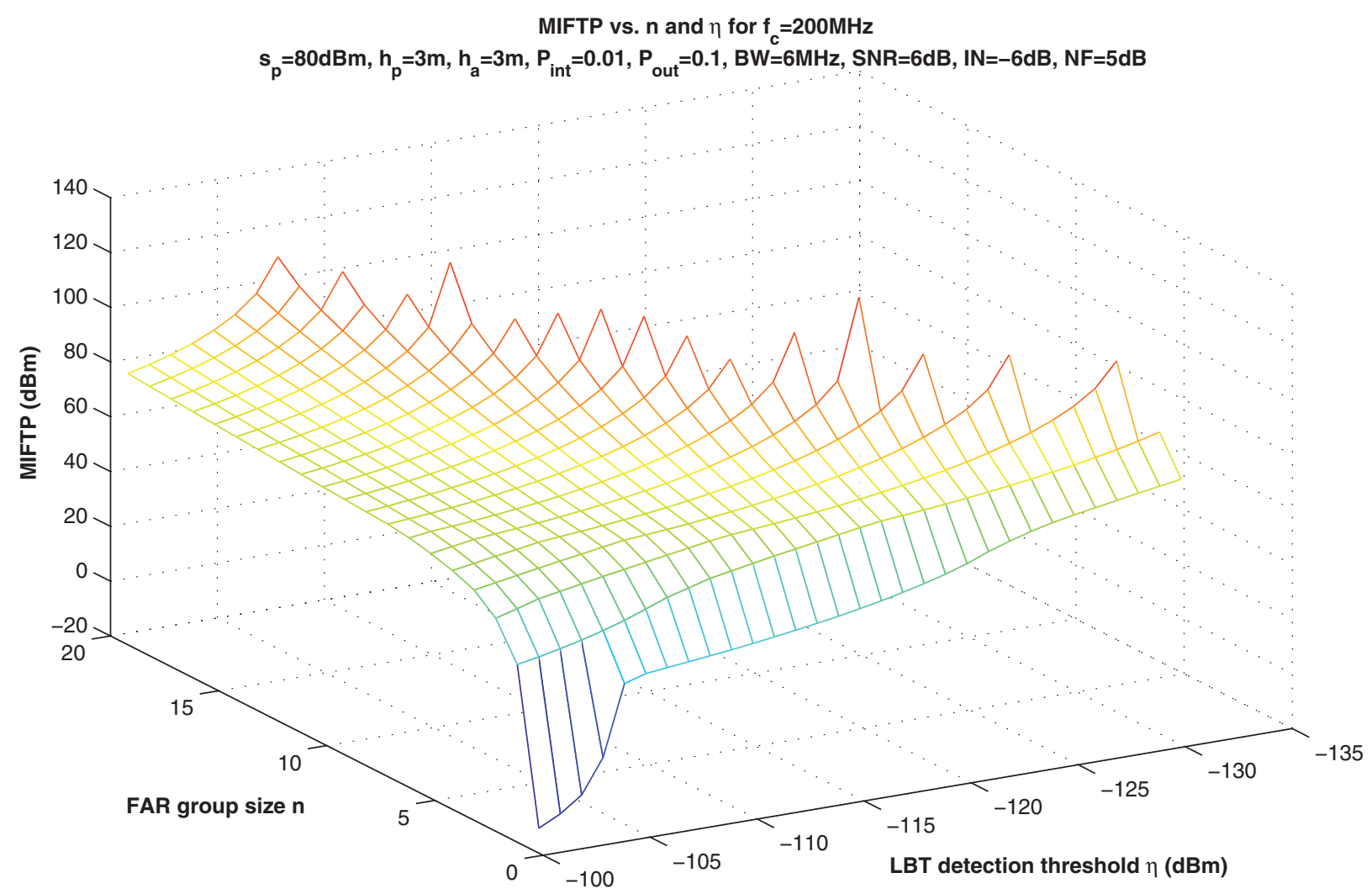

Figure 13. MIFTP versus detection threshold and FAR group size for collaborative sensing at $f_{c}=2 \mathrm{GHz}$

\section{ACKNOWLEDGEMENTS}

This work was supported in part by the National Science foundation under Grant CNS-0520151, and also by the Defense Advanced Research Projects Agency and the Air Force under the XG Program, Contract FA8750-05-c150 (Approved for Public Release, Distribution.)

\section{REFERENCES}

1. McHenry M. Frequency agile spectrum access technologies. In Proceedings of the FCC Workshop on Cognitive Radio, May 2003.

2. Staple G, Werbach K. The end of spectrum scarcity. IEEE Spectrum 2004; 41: 48-52.

3. Haykin S. Cognitive radio: brain-empowered wireless communications. IEEE Journal of Selected Areas in Communication 2005; 23: 201-220.

4. Mitola J, Maguire GQ. Cognitive radio: making software radios more personal. IEEE Personal Communications 1999; 6(August): 13-18.

5. Leu AE, Steadman K, McHenry M, Bates J. Ultra sensitive TV detector measurements. In Proceedings of the IEEE International Symposium on New Frontiers in Dynamic Spectrum Access Networks (DySPAN), November 2005; 30-36.

6. McHenry M. The probe spectrum access method. In Proceedings of the IEEE International Symposium on New Frontiers in Dynamic Spectrum Access Networks (DySPAN), November 2005; 346-351.

7. Fong TK, Henry PS, Leung KK, Qiu X, Shankaranarayanan NK. Radio resource allocation in fixed broadband wireless networks. IEEE Transactions on Communications 1998; 46(6): 806-818.

8. Gudmundson M. Correlation model for shadow fading in mobile radio systems. Electronic Letters, 1991; 27: 2145-2146. 
9. Leu AE, Mark BL. Modeling and analysis of fast handoff algorithm for microcellular networks. In Proceedings of the IEEE International Symposium on Modeling, Analysis and Simulation of Computer and Telecommunication Systems (MASCOTS), October 2002; 321-328.

10. Raychaudhuri $D$, Jing $X$. A spectrum etiquette protocol for efficient coordination of radio devices in unlicensed bands. In Proceedings of PIMRC, Beijing, China, 2003; 172-176.

11. Popescu O, Rose C. Waterfilling may not good neighbors make. In Proceedings of IEEE Globecom, Dallas, TX, November 2004; 1766-1770.

12. Lustgarten MN, Madsen JA. An empirical propagation model (EPM-73). IEEE Transactions on Electromagnetic Compatibility 1977; 19: 301-309.

13. Longley AG, Rice PL. Prediction of tropospheric transmission loss over irregular terrain, a computer method1968. ESSA 79-ITSS, US Department of Commerce, Office of Telecommunications Boulder, CO, July, 1968.

14. Longley AG, Hufford GH, Reasoner R, Montgomery J. A statistical propagation model for the improved interference prediction model. ESSA OT-TM 67, US Department of Commerce, Office of Telecommunications, Boulder, CO, December 1971.

15. Adawi NS, Bertoni HL, Child JR, Daniel WA, Dettra JE, Eckert RP, Flath EH, Forrest RT. Coverage prediction for mobile radio systems operating in the $800 / 900 \mathrm{MHz}$ frequency range. IEEE Transactions on Vehicular Technology 1988; 37: 3-72.

\section{AUTHOR'S BIOGRAPHY}

Alexe E. Leu received the B.S. and M.S. degrees in Electronics and Telecommunications from Politehnica University of Bucharest, Romania, in 1995 and 1996, respectively and the Ph.D. degree in Electrical and Computer Engineering from George Mason University, Fairfax, VA in 2003. From 1999 to 2001, he was a design engineer in the R\&D Department at LCC International Inc, McLean, VA. Since 2003 he has been a Senior Research Engineer at Shared Spectrum Company, McLean, VA. His research interests are in cognitive radio; design, modeling, and performance evaluation of wireless network architectures and protocols; integrating spatial and temporal processing for mobile networks.

Mark A. McHenry received the B.S. degree in Engineering and Applied Science from the California Institute of Technology in 1980, the M.S. degree in Electrical Engineering from the University of Colorado in 1984 and the Ph.D. degree in Electrical Engineering from Stanford University in 1988. He was a Senior Engineer at Northrop Advanced Systems, Pico Rivera, CA, from 1982-1985, and a Senior Research Engineer at SRI International, Menlo Park, CA, from 1988-1996. From 1996-2000, he was a Program Manager at DARPA, Arlington, VA, where he managed several programs in the areas of wireless communications, imaging, and tactical aircraft landing. He was Co-founder and Chief Technical Officer of San Diego Research Center from 2001-2003. Since 2000, he has been President of the Shared Spectrum Company, McLean, VA, which is engaged in the research and development of automated spectrum management technologies and highly dynamic tuners. He received Office of Secretary of Defense Awards in 1997 and 2000 for Outstanding Achievement and Exceptional Public Service, respectively.

Brian L. Mark received the B.A.Sc. degree in computer engineering with an option in mathematics from the University of Waterloo, Canada, in 1991 and a Ph.D. in electrical engineering from Princeton University, Princeton, NJ, in 1995. He was a Research Staff Member at the C\&C Research Laboratories, NEC USA, from 1995 to 1999. In 1999, he was on part-time leave from NEC as a visiting researcher at Ecole Nationale Superieure des Telecommunications in Paris, France. In 2000, he joined the Dept. of Electrical and Computer Engineering at George Mason University, where he is currently an Associate Professor. His main research interests lie broadly in the design, modeling, and analysis of communication systems, computer systems, and communication networks. He was co-recipient of the best conference paper award for IEEE Infocom'97. He received a National Science Foundation CAREER Award in 2002. 